\title{
Managing Heterogeneous Data Flows in Wireless Sensor Networks Using a 'Split Personality' Mote Platform
}

\author{
Danny Hughes ${ }^{1}$, Mickaël Daudé ${ }^{2}$, Geoff Coulson ${ }^{1}$, Gordon Blair ${ }^{1}$, \\ Paul Smith ${ }^{3}$, Keith Beven ${ }^{3}$, Wlodek Tych ${ }^{3}$ \\ ${ }^{1}$ Computing Department, InfoLab21, South Drive, Lancaster University, Lancaster, UK. LA1 4WA \\ \{danny,geoff, gordon\} @ comp.lancs.ac.uk \\ ${ }^{2}$ INP ENSEEIHT, Toulouse, France. 31071 \\ mickael.daude@etu.enseeiht.fr \\ ${ }^{3}$ Environmental Science Department, Lancaster University, Lancaster, UK. LA1 4YQ \\ $\{k$. beven, p.j.smith,w.tych\}@lancaster.ac.uk
}

\begin{abstract}
Remote sensing applications are increasingly making use of sensors that generate demanding data flows (e.g. digital imaging devices). This paper presents the design of GridStix 1.5, a novel wireless sensor network platform that offers alternative high-performance and low-power 'personalities'. This two-tier approach allows GridStix 1.5 to provide power efficient support for sensors which produce simple data flows, and rich computational and network support for more demanding sensor types. Our approach is evaluated in the context of a deployed flood monitoring and warning system.
\end{abstract}

\section{Introduction}

Nodes in wireless sensor networks (WSNs) are increasingly required to support complex data flows and in-network computation [1] [11]. Examples of such complex sensor nodes are digital cameras, audio sensors and sensors based on spectrum analysis. In the flood monitoring scenario featured in this paper (see section 2), significant computing and networking resources are employed to support image-based flow measurement and on-site flood modeling. But our system also supports more 'common-place' WSN functionality such as relaying readings from pressure transducers and conductivity sensors to a GSM uplink [1].

Image based flow measurement and on-site flood modeling require support that is not typically present in WSN environments: specifically, network overlay support for data-flows between motes, support for sporadic high-bandwidth communications, and innetwork computation. These requirements are discussed in more detail in section 2.

Our previous-generation GridStix platform supported flood warning and prediction using a combination of our Open Overlays middleware platform [2] and an extended version of the GumStix [3] embedded computing platform. Unfortunately, while this platform was capable of meeting significant computational and networking demands, it did so at the expense of power consumption. This paper presents the GridStix 1.5 platform which addresses the energy consumption shortcomings of GridStix v1 by providing two discrete 'personalities': a power-saving personality suitable for simple data flows and a more capable high performance personality suitable for supporting complex data flows. In the context of the highperformance personality, the Open Overlays platform provides rich data flow support, while in the context of the low power personality; Open Overlays is used to support a more basic configuration.

The remainder of this paper is structured as follows: Section 2 discusses system requirements of nextgeneration sensor nodes in the context of complex data flows and in-network computation; Section 3 describes the GridStix 1.5 platform; Section 4 describes the deployment environment and initial evaluation; Section 5 provides context on related work; and Section 6 offers our conclusions and proposes directions for future research.

\section{System Requirements}

Our flood monitoring system [1] supports three distinct processes that impose quite different system requirements: i) reporting of sensor data; ii) in-network computational flood modeling; and iii) image based flow measurement. The computational and data-flow requirements of these processes are discussed in Sections 2.1 and 2.2 respectively. Reporting of sensor readings occurs continually while in-network computational flood modeling and image-based flow 
measurement are performed only when dictated by environmental conditions [1].

\subsection{Requirements of Application Data Flows}

The networking requirements imposed by the reporting of depth readings, in-network computational flood modeling and image-based flow analysis are as follows:

Reporting of depth readings Our system sends data from pressure-based depth sensors to a GSM uplink for dissemination off-site. Each sensor reading comprises a 1 byte node identifier, a time-stamp and two 12 bit ADC readings, giving a total size of 5 bytes per sensor reading. Pressure sensors are sampled at intervals of five minutes and during sampling, one sensor reading is taken per second for a period of 20 seconds. Thus depth sensors generate a predictable data flow rate of 100 bytes at intervals of five minutes which must be relayed from 15 sensor nodes to a single gateway. Reporting of depth readings occurs during non-flood conditions and generates a classic low bandwidth many-to-one data flow from sensor nodes to the GSM gateway. This functionality is implemented by a spanning tree implementation running on the $433 \mathrm{MHz}$ radios [4]. Due to the ability of flood models to deal with sporadic and imperfect data, only a small cache of sensor readings is maintained on each node with comprehensive logging / archiving being performed offsite.

In-network computational flood modeling Our system also supports in-network computational flood modeling, which allows the system to provide flood warning functionality without the necessity of connection to off-site computational facilities [1]. The necessary per-node computation requires data (this is a sequence of sensor readings, along with predictions from the computation on the remote node) from a small number of other nodes. The flood models can tolerate latency of multiple seconds and require a maximum throughput of no more than 10kbps [5]. In-network flood modeling occurs when flooding is predicted and generates a bi-directional data flow between nodes located in geographically relevant locations. As the relevance of remote nodes is dependent upon the output of the flood model, these data flows may change dynamically. This data flow is served by an ad-hoc networking infrastructure implemented over the low bandwidth $433 \mathrm{MHz}$ radios. In order to ensure resiliency against data loss during critical flood periods, each node sends its last three updates along with the current update.

Image-based flow measurement Our system also supports image-based flow measurement. This is an emerging technique which uses cheap off the shelf digital cameras to measure flow rates [1]. Image-based flow measurement requires the dissemination of multiple high resolution images among sensor nodes which must be distributed in a timely fashion. This requires up to $1 \mathrm{MBPS}$ of bandwidth and thus cannot be supported by low power radio hardware. Image based flow measurement occurs during periods of flooding and high flow rates. This generates a bi-directional high bandwidth many-to-one data flow between the nodes equipped with digital imaging hardware and nodes participating in image analysis. This is served by adhoc 802.11 b networking. In terms of data storage, the camera-equipped nodes maintains a large cache of recent image files, while each remote node participating in distributed image analysis will receive a set of up to $4 \mathrm{MB}$ of images.

\subsection{Computation Requirements}

Each of the three identified areas of system functionality also has distinct computational requirements. Transmission of sensor readings requires very little computational power and can easily be supported on a low power microcontroller such as the Atmel ATMEGA128 MCU [6]. In-network computational flood modeling and image-based flow measurement require significantly greater computational power than is available on low power microcontrollers. However, these processes can be executed in a timely fashion on more powerful embedded processors such as the Intel PXA255 [3]. In summary, reporting of depth readings has low computation and network requirements. On-site flood modeling has high computation but low network requirements. Image based flow analysis has high computation and network requirements.

The following section describes how the GridStix 1.5 platform uses adaptation to effectively manage the heterogeneous data-flow requirements of our system.

\section{The GridStix 1.5 Platform}

The original GridStix platform is described in detail elsewhere [1]. In brief, each GridStix v1 node consists of a GumStix embedded computer with a $400 \mathrm{MHz}$ Intel XScale PXA255 CPU, 64Mb of RAM and 16Mb of flash memory and 802.11b [3]. Each node runs the above mentioned Open Overlays middleware. This is a light-weight component based WSN software platform that supports run-time reconfiguration [2] and provides rich networking support from the MAC level to the application level. Unfortunately, while GridStix v1 supported the requirements outlined in section 2 , it did so at the expense of high power consumption which significantly reduced the duration of deployments. 
The GridStix 1.5 platform implements a 'lowpower' personality which allows the node to support power-efficient transmission of sensor readings during quiescent conditions, and also support for more complex applications as demanded by environmental conditions. As discussed, high computational and networking performance are only required for innetwork computational flood modeling and imagebased flow analysis, which only execute during flood conditions. The hardware and software extensions which comprise GridStix 1.5 are described in sections 3.1 and 3.2 respectively.

\subsection{The GridStix 1.5 Hardware Platform}

GridStix 1.5 adds a custom-built low power mote platform to the original GridStix platform (the 'highperformance' personality). The mote platform comprises an Atmel ATMEGA128 [5] with 4KB RAM, low power $433 \mathrm{MHz}$ radio module [4], 512KB flash memory and battery backed real-time clock. The low power mote is linked to the GridStix via the I2C bus and can operate in either 'master' or 'slave' mode. In slave mode, the low-power mote acts as an expansion board for the GridStix, adding low power radio, flash memory and real-time clock. In master mode, the GridStix is deactivated and the low-power mote implements basic wireless sensor network functionality.

\subsection{The GridStix 1.5 Software Platform}

The low power mote implements basic sensing and networking functionality which may be easily configured from the 'high-performance' personality.

In terms of sensing, developers can schedule the logging and transmission of sensor readings. In addition, they may specify simple alarm conditions which are used to activate the high-power personality. For example, they may specify that if depth increases at a certain rate (i.e., flooding may be imminent) then the high-performance personality should be activated.

In terms of networking, the low-power mote provides a self organizing and self healing spanning tree overlay implementation suitable for supporting the data flow requirements of reporting depth readings i.e. a low-bandwidth many-to-one relationship. The structure of this spanning tree may also be configured from the high-performance personality to best suit environmental conditions as discussed in [2]. In 'slave' mode, GridStix 1.5 provides support for the on site flood modeling data flow - i.e. low bandwidth ad-hoc networking. Finally, to provide support for the imagebased flow analysis data flow, - i.e. high bandwidth adhoc communication, GridStix 1.5 switch to using $802.11 \mathrm{~b}$ ad-hoc networking.
Configuration of the low power personality is achieved by writing to the low power mote's flash memory, which is accessible via the shared I2C bus. Configuration functionality is itself wrapped in a standard Open Overlays component framework [2], allowing developers to configure the low-power personality using standard Open Overlays interfaces. Similarly, in slave mode, developers have access to peripherals using standard Open Overlays interfaces.

\subsection{Exploiting Split Personalities}

The two personalities are appropriately exploited by matching the behaviour of the system to best suit the demands of the data flow conferred by available resources and conditions. For example, during quiescent conditions, when water depth and flow-rate are within normal bounds, there is no benefit in running flood modeling and image-based flow measurement. During such conditions, GridStix 1.5 enter the lowpower personality to conserve battery life. However, when conditions indicate that flooding may occur, GridStix 1.5 enter the high-performance personality (as triggered by the low power mote), thereby providing sufficient computational and networking support for innetwork computational flood modeling and imagebased flow measurement. Similarly, if mote battery levels run low, the system can scale back to the lowpower personality, avoiding total system failure.

\section{Deployment and Initial Evaluation}

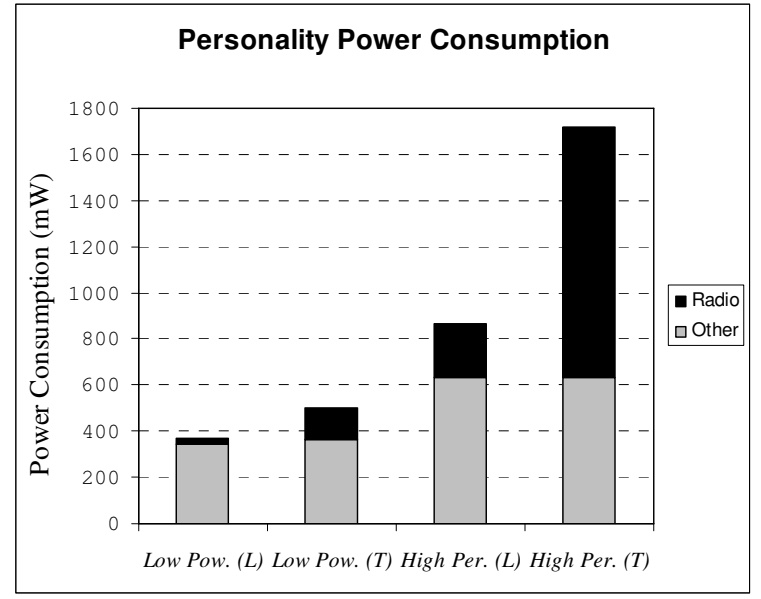

Figure 1 - Power Consumption of Each Personality

We are currently in the process of re-deploying a network of GridStix 1.5 on the River Dee in North Wales and we expect deployment to be completed by late spring 2008. While this deployment in ongoing we have evaluated the GridStix 1.5 platform in the lab in terms of functionality and power consumption. Specifically, figure 1 compares the power consumption 
of the high-performance and low-power GridStix 1.5 personalities while listening (L) and transmitting (T). The power draw of the radio is shown in black, while other power draw (i.e. CPU, memory etc.) is shown in grey.

As can be seen, the low-power personality consumes significantly less power than the highperformance personality. Power consumption is $57 \%$ lower while listening and $71 \%$ lower during message transmission. This allows system deployments to be radically extended during periods when power is scarce or when support for complex data flows is unnecessary.

\section{Related Work}

A number of projects have tackled the problem of providing flood support using WSN technology. The Floodnet project [9] uses a platform similar to GridStix v1 (an XScale CPU with $802.11 \mathrm{~b}$ networking) to implement flood monitoring on a tidal river in South East England, though this system supports only simple data flows, providing no support for in-network computation. The Hydrowatch project uses low power Telos motes to implement river monitoring in the Sierra Mountains in northern California [10]. This project is currently focused on supporting the planning of micro-solar installations to support environmental monitoring WSNs. The mote platform used in Hydrowatch does not have sufficient resources to support in-network computation, though the project does offer support for more complex data flows through the use of 6LowPAN on-site networking [8].

The approach of separating the concerns of networking and sensing from application processing is also used in Dust Networks 'Smart Mesh' products [6] which provide reliable mesh networking and basic sensing functionality with the expectation that developers will add their own application processor; while the GainSpan GS1010 [7] provides separate application and network processors. Such a separation allows power-hungry application processors to be activated only when needed. We are currently evaluating the Dust Networks SmartMesh-XT 2135 alongside the GridStix 1.5 low-power personality for providing low power networking support.

\section{Conclusions and Future Research}

We have described a WSN node architecture that employs two personalities: a low power one and high performance one. We have also discussed in outline how the new capabilities enabled by this platform are controlled and managed by our Open Overlays middleware. Based on this combination of hardware and software, we expect that our flood system deployments can survive for significantly longer durations while offering support that is specifically tailored for the diverse data flows of the flood warning application.

Our future research will initially focus upon deploying the GridStix 1.5 flood monitoring platform at the new site on the River Dee. The GridStix 1.5 platform will then be evaluated more thoroughly in this deployed environment. As well as evaluating low-level system functionality, we are particularly interested in evaluating the role of adaptation in improving system performance. This includes adaptation between personalities as discussed in this paper, as well as more fine grained adaptation such as the adaptation of networking behaviour [2].

\section{References}

[1] Hughes D., Greenwood P., Coulson G., Blair G., Pappenberger F., Smith P., Beven K., An Intelligent and Adaptable Flood Monitoring and Warning System, in the proceedings of the 5th UK E-Science All Hands Meeting (AHM'06), Nottingham, UK, September 2006.

[2] Grace P., Hughes D., Porter B., Blair G., Coulson G., Taiani F., Experiences with Open Overlays: A Middleware Approach to Network Heterogeneity, in the proceedings of the European Conference on Computer Systems (EuroSys'08), Glasgow, UK, March 2008.

[3] GumStix Embedded Computing Platform, http://gumstix.com/, February 2008.

[4] Low Power Radio Solutions, Easy Radio Data Sheet, available online at: www.radiomodules.com/products/radiodatamods/datasheets/er-datasheet2.3-sept-05.pdf, 2005.

[5] Atmel Corporation, ATMEGA128 Microcontroller Data Sheet, available online at: http://www.atmel.com/dyn/resources/prod_documents/doc2467. pdf, 2008.

[6] GainSpan, GS1010 Ultra Low-Power Wireless System-OnChip Data Sheet, available online at: http://www.gainspan.com/Docs/GS1010_SoC_Product_Brief.pd f, 2008 .

[7] Dust Networks, SmartMesh-XT 2135 Data Sheet, available online at: http://www.dustnetworks.com/docs/M2135.pdf, 2008.

[8] Montenegro G., Kushalnagar N., Hui J., Culler D., RFC 4944 - IPv6 over Low Power Wireless Personal Area Networks (6LowPAN) RFC, available online at: http://www.ietf.org/rfc/rfc4944.txt

[9] DeRoure D., "Improving Flood Warning times using Pervasive and Grid Computing", available online at: http://envisense.org/floodnet/ingenia/ingenia.htm.

[10] Taneja J., Jeong J., and Culler D., Design, Modeling, and Capacity Planning for Micro-Solar Power Sensor Networks, in the proceedings of the Seventh International Conference on Information Processing in Sensor Networks (SPOTS '08), April 2008.

[11] Rahimi M., Baer R., Iroezi O., Warrior J., Estrin D., Srivastava m., Cyclops: in situ image sensing and interpretation in wireless sensor networks, in the proceedings of the $3^{\text {rd }}$ Conference On Embedded Networked Sensor Systems (Sensys 2005), San Diego, California, USA, pp 192 - 204. 